\title{
The Moon Illusion Explained by Visual Motion Perception
}

\author{
Ahmad Yousef ${ }^{1}$ \\ ${ }^{1}$ School of Computational Science and Engineering, McMaster University, Hamilton, Ontario, Canada \\ *Correspondence: mohamas2@mcmaster.ca
}

\begin{abstract}
We designed two virtual space missions to the moon, namely, two virtual rockets were launched to the horizon moon and to the zenith moon. These virtual rockets carry exactly the same physical characteristics (length, width, contrast, and speed). Our observers had noticed when the rocket travelling horizontally to the horizon moon, it appears to move way slower; deceleration that may trigger the brain to pull the moon towards the observers to compensate the delay of the aforementioned virtual space mission. Introduction

The Moon illusion could be a human visual illusion that causes the horizon moon to appear much bigger than the zenith moon. Shockingly, the moon near the horizon is further than the moon in the zenith zone from the frames of references (humans), namely, the distance between the frame of reference and the horizon moon is greater about the radius of the earth than the distance between the same reference and the zenith moon. In another word, the aforementioned statement should add further complication to understand the moon illusion. The reason behind the moon illusion is thought to be due atmospheric reflections, but due to lack of convincing physical reasoning, scientists started to skeptically believe in apparent distance hypothesis,
\end{abstract} see references 1 and 2 . Other scientists argue that the moon illusion is due to how the brain

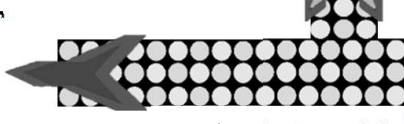
configures depth perception by scaling To Horizon Moon observed that the horizontal rockets exhibits much slower perceived speed than the virtual vertical rockets. We therefore had decided to run three experiments to examine this observation in twelve observers. The first experiment is to quantifyingly compare between the perceived speed of the horizontal rockets versus the perceived speed of the virtual vertical rockets. The second experiment is to prove that Wundt horizontal-vertical illusion not only works for lines but for planar shapes, namely, the aforementioned rockets. We generally notice that the vertical rockets are longer but thinner than the virtual horizontal rockets, as if the human visual system compressed the vertical rockets from the sides towards its centroid; compression that makes the width of the rockets look thinner, but compensates that loss of the overall area in adding greater length. Intuitively speaking, and in accordance to the Newtonian mechanics, the second experiment may hypothesize that the vertical rockets gained higher perceived speed to compensate the greater perceived length, however, the obtained statistics from our behavioral data have refused to comply to Newtonian mechanics, further information will be provided in the discussion section. No violation against the general conscious theory of relativity, the third experiment concluded. The general conscious of everything with respect to the horopter, see reference 3; and others rely on the projective consciousness model, see reference 4. Many scientists, however, think that Ebbinghaus illusion may generously explain the moon illusion, and this interpretation might resolve some scientific debates. see reference 5 . We, however, think that there are other physical and psychophysical theories that may also explain the moon illusion. We primarily had

relativity talks about how spatial attention has outstanding capability to dilates time and length, see reference 7 . We had previously studied the aforementioned theory exclusively on horizontal visual motion stimuli, in this study, however, we included vertical visual motion stimulus to inform the readers about the validity of the aforementioned theory, regardless the directive presentation.

\section{Materials \& Methods}

Twelve human subjects, with dilated pupils, were recruited to participate in the three experiments. 
Each stimulus subtends 12.4 DVA in length, and 1.72 DVA in width for the first two experiments. For finetuning purposes, each human subject has to undergo into three trials for each experiment. Each trial in the first experiment presents the virtual horizontal rockets for 6 seconds, then the vertical ones for another 6 seconds and then the previous process is repeated to complete one trial. The estimations of the length and the thickness of the rockets are achieved in the second experiment using the same visual stimuli, but in further trials. The third experiment, however, has used exactly the same (Green) horizontal visual motion stimulus offered in reference 7 ; a $90^{\circ}$ rotation were applied to the aforementioned stimulus to test whether the general conscious of relativity is still valid for vertical visual motion perception. For each stimulus, the human subjects had been tested for three times, and their task to give quantitative subjective judgments of how spatial attention can decelerate the velocity and dilate the length.

Data Collection \& Structural Analysis

The behavioral data were collected manually, and then averaged among the human subjects to estimate the statistical values, namely, the average and the standard error of the mean of the different aforementioned psychometrics for the three experiments are then compared to frames of references.

Results; Three Experiments

First Experiment:

The virtual vertical rockets have greater speed than the horizontal ones with mean value $\approx 0.41$, and SEM $\approx$ 0.046 .

Second Experiment:

The virtual vertical rocket has greater length than the horizontal ones with mean value $\approx 0.28$, and SEM $\approx$ 0.053; and it has thinner width than the horizontal ones with reduced mean value $\approx 0.17$, and $S E M \approx$ 0.042;

Third Experiment:

Horizontal visual motion perception: Spatial attention dilates the time with mean value $\approx 0.74$, and SEM $\approx$ 0.26 ; and it dilates the length with mean value $\approx 0.28$, and $S E M \approx 0.14$

Vertical visual motion perception: Spatial attention dilates the time with mean value $\approx 0.89$, and SEM $\approx$ 0.31 ; and it dilates the length with mean value $\approx 0.35$, and $S E M \approx 0.16$.

\section{Conclusion and Discussion}

The moon illusion could be explained by the perceived speed of vertical versus horizontal apparent motion. Seemingly, Wundt horizontal-vertical illusion plays a role on how the human subjects perceives the visual motion, however, our collected behavioral data did not show perfect fitting between the perceived length and speed to be consistent with the Newtonian mechanisms, namely, the secret of the moon illusion will remain debatable for a further notice. The validation of the general conscious of relativity for vertical motion, however, should remove the readers' skepticism about contradictions between our initial experiments in this study, and the general conscious theory of relativity. The validation of the theory, however, triggers several exploratory questions, namely, profound studies of the mechanisms of the spontaneous (vertical versus horizontal) saccadic eye movements, in addition to, several retinal studies (vertical versus horizontal retinal peripheries) become extremely essential.

\section{Acknowledgement}

We would like to sincerely thank Edward Tehovnik for his generous feedbacks and assistance.

\section{Essential Notifications}

Readers should have direct access to the stimuli, see reference 8. Advisably, download the videos for the best quality. For rapid validation process, have the test with dilated pupil. and watch the videos in moderate environment; namely, fifty percent of the monitor's max. brightness. Caffeine intake also dilate the pupil, in case of pinpoint pupils, see reference 9. Scholars with professional eye tracker, and brain imaging facilitations are welcome to collaborate in challenging investigations. Enquiries may be sent to the author. Transactional References

[1] Ross, Helen; Plug, Cornelis (2002). The mystery of the moon illusion. Oxford University Press.

[2] Kaufman, etal. (2007). "Perceptual distance and the moon illusion". Spatial Vision.

[3] Edward Tehovnik (2020): Personal communications in ResearchGate platform.

[4] D. Rudrauf, D. Bennequin \& K. Williford, The Moon Illusion explained by the Projective Consciousness Model. ArXiv, 2018.

[5] Restle, (1970). "Moon Illusion Explained on the Basis of Relative Size". Science.

[6] Wundt W. (1858). "Vertical-horizontal illusion", Published online Wikipedia, 2015.

[7] Yousef, A. 2019. "Spatial Attention Dilates Time and Length." PsyArXiv. doi:10.31234/osf.io/qazj9.

[8] You may find the stimuli in the following link: https://drive.google.com/drive/folders/1d4PfjQo7ols45gHbt $\mathrm{H}$ tOMRvvYPSaqKXO

[9] Abokyi, etal.,(2017). Caffeine intake is associated with pupil dilation and enhanced accommodation. Eye Journal. 